\title{
EMDR Therapy's Efficacy in the Treatment of Pain
}

\author{
Jonas Tesarz \\ University Hospital Heidelberg, Heidelberg, Germany \\ Manon Wicking \\ Kathrin Bernardy \\ Ruhr University, Bochum, Germany \\ Günter H. Seidler \\ University Hospital Heidelberg
}

\begin{abstract}
Chronic pain is the most common global cause of functional and quality of life limitations. Although there are many effective therapies for the treatment of acute pain, chronic pain is often unsatisfactory. Against this background, there is currently an urgent need to develop innovative therapies that enable more efficient pain relief. Psychosocial factors play an important role in the development and persistence of chronic pain. Especially in patients with high levels of emotional stress, significant anxiety, or relevant psychological comorbidity, classical pain therapy approaches often fail. This is in line with the results of recent pain research, which has shown that dysfunctions in emotion processing have a significant influence on the persistence of pain symptoms. The recognition that pain can become chronic through maladaptive emotional processing forms the pathophysiological basis for the application of eye movement desensitization and reprocessing (EMDR) in the treatment of chronic pain. In this sense, EMDR can be used as an established method for desensitizing and processing of emotional distress from trauma therapy specifically for processing emotional stress in patients with chronic pain. Against this background, it is not surprising that the implementation of EMDR for patients with chronic pain is expanding. However, the increasing clinical use of EMDR in the treatment of chronic pain has also led to a reputation to test the efficacy of EMDR in pain management through randomized clinical trials. In addition to numerous case control studies, there are now also six randomized controlled clinical trials available that demonstrate the efficacy and safety of EMDR in the treatment of different pain conditions. However, in order to overcome several methodological limitations, large multicenter studies are needed to confirm the results.
\end{abstract}

Keywords: eye movement desensitization and reprocessing (EMDR); pain; treatment; narrative review

C hronic pain is one of the most serious health problems worldwide (Buchbinder et al., 2018). It is estimated that more than $20 \%$ of the world's adult population suffer from chronic pain, and every 10th person is diagnosed with chronic pain every year (International Association for the Study of Pain, 2012). Chronic pain is therefore one of the most common causes of disability and quality-of-life impairments worldwide (GBD 2016 Disease and Injury Incidence and Prevalence Collaborators, 2017).
In a considerable number of patients, the pain persists despite adequate treatment of the underlying disease. This is especially the case in patients with comorbid mental disorders and in patients where psychological factors play a major role. These patients are complex, cost-intensive, and represent a major challenge for clinical care. Various therapies are available, and a multimodal treatment principle based on a biopsychosocial model is generally recommended. However, the treatment of chronic pain is usually difficult 
and the treatment outcomes unsatisfactory (Williams, Eccleston, \& Morley, 2012), with small to moderate effect sizes. While some patients benefit significantly from generic treatment approaches, others do not benefit or even incur adverse effects. This is especially true when comorbid mental disorders such as anxiety, stress-related disorders, or depression are present, and psychological factors play a major role in the chronification. However, especially in these patients the application of eye movement desensitization and reprocessing (EMDR) seems to be highly effective in reducing pain symptoms. Whether EMDR is an effective intervention here and in which pain patients the method works is an important question for both EMDR practice and research. In this brief narrative review, the current scientific evidence on the efficacy of EMDR in the treatment of chronic pain is presented.

\section{Why EMDR for the Treatment of Pain?}

The treatment of pain represents a promising and recently growing field for the use of EMDR therapy. Psychotherapeutic care of patients with pain has long focused on their dysfunctional coping strategies and maladaptive behavior patterns. However, the significant influence of stressful life events and/or emotional stress on pain perception and central pain processing has been neglected (Tesarz et al., 2015; Tesarz et al., 2016). In addition to the purely sensory dimension, physical pain usually contains a substantial emotional dimension. This emotional dimension not only determines the levels of intensity and/or stress with which pain is experienced, but it can also contribute significantly to the maintenance - that is, chronificationof the pain symptomatology. Recent neurobiological studies have shown that the process of pain chronicity in the brain leads to a shift away from classical pain-processing regions towardemotional neural networks (Hashmi et al., 2013). This "emotional" shift is held responsible for the fact that pain, similar to "flashbacks" in posttraumatic stress disorders, settles in and is unable to disappear.

The understanding that the process of pain chronicity is closely linked to maladaptive emotional processing forms the pathophysiological basis for the application of EMDR therapy in the treatment of chronic pain: an established method for processing emotionally stressful experiences in trauma therapy can be specifically used for processing emotional stress in patients with chronic pain, thereby processing and resolving this dysfunctional "emotional shift." In addition to the EMDR-specific element of "desensitization and reprocessing," EMDR contains numerous other EMDR-unspecific pain-relieving therapeutic elements (exposure, relaxation and hypnosis techniques, improvement of coping skills, cognitive restructuring, etc.) that can be adapted to the individual needs of the patient due to the patient-centered character of EMDR. Against this background, it is not surprising that EMDR is used increasingly to treat patients with chronic pain (Tesarz et al., 2014; Tesarz, Seidler, $\&$ Eich, 2015). In addition to a large number of uncontrolled observational studies, there is now also a growing number of randomized controlled trials that have investigated the efficacy of EMDR in pain therapy.

\section{Scientific Evidence for the Use of EMDR in the Treatment of Pain}

In 2014, a systematic review was able to identify a total of 2 controlled trials and 10 observational studies (Tesarz et al., 2014). Since then, 4 more randomized controlled trials have been published. Thus, there are currently a total of six randomized controlled trials that have investigated the clinical efficacy of EMDR in pain therapy. An examination of the case and controlled studies reveals promising results, especially for phantom pain (De Roos et al., 2010; Schneider, Hofmann, Rost, \& Shapiro, 2008; Rostaminejad, Behnammoghadam, Rostaminejad, Behnammoghadam, \& Bashti, 2017; Wilensky, 2006), headache (Konuk, Epözdemir, Atçeken, Aydin, \& Yurtsever, 2011; Marcus, 2008; Mazzola et al., 2009), acute postoperative pain (Maroufi, Zamani, Izadikhah, Marofi, \& O'Connor, 2016), and chronic musculoskeletal disorders (Allen, 2004; Gerhardt et al., 2016; Grant \& Threlfo, 2002; Nia, Afrasiabifar, \& Behnammoghadam, 2018). These studies consistently showed a significant improvement in pain intensity, with overall high effect sizes following EMDR interventions. The effect sizes varied significantly depending on the disease entity and duration of treatment. With regard to the sustainability of the therapy effects achieved, the follow-up studies showed stable effects or even further improvements, which provided preliminary evidence that EMDR appears to be sustainably effective. However, whether specific mechanisms are responsible for the effects remains unclear. Possible unspecific effects like increased attention of healthcare professionals, distraction, or relaxation might have driven the results. Note that methodological limitations within and between studies require a cautious interpretation of these impressive results. See Table 1. 


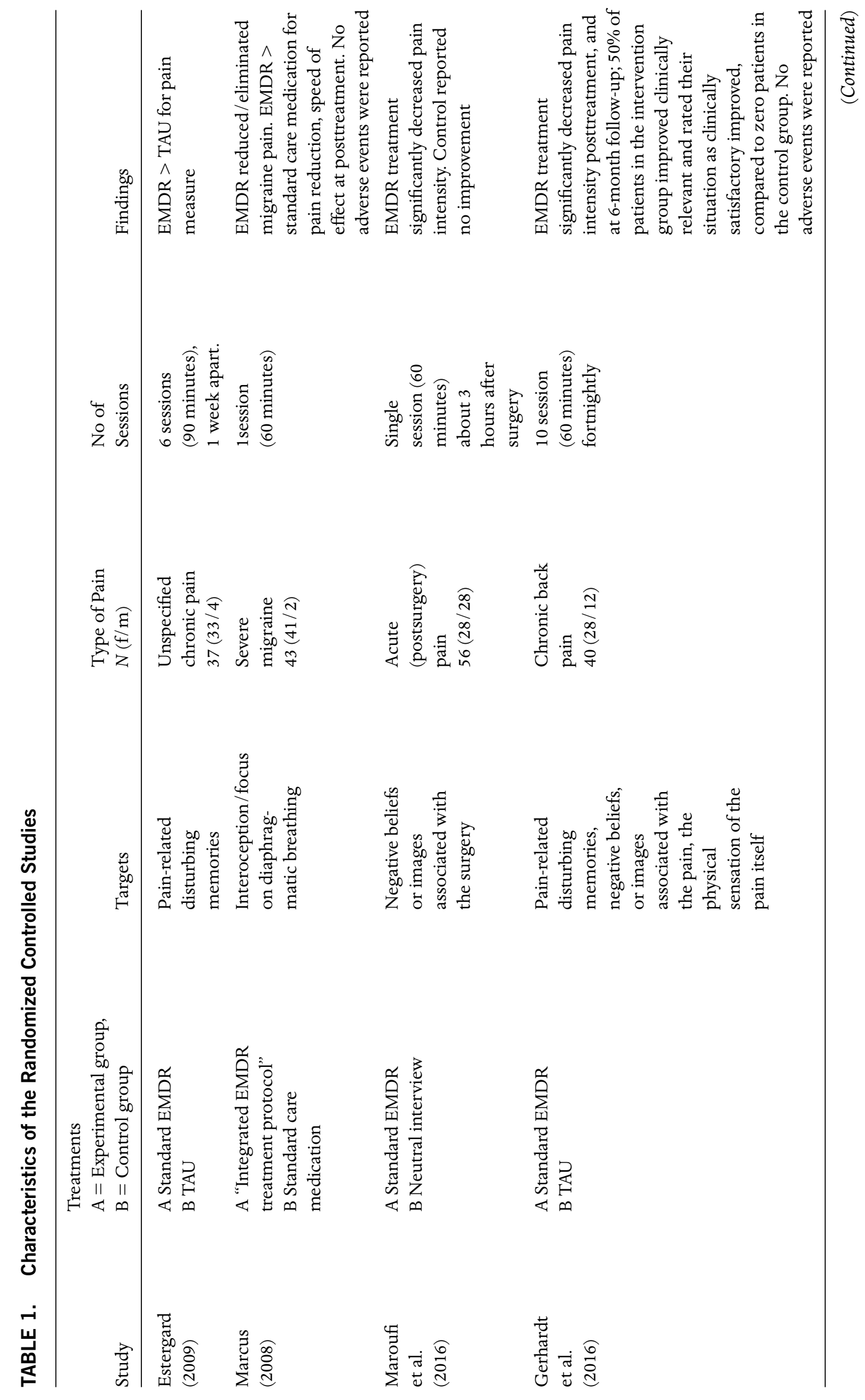




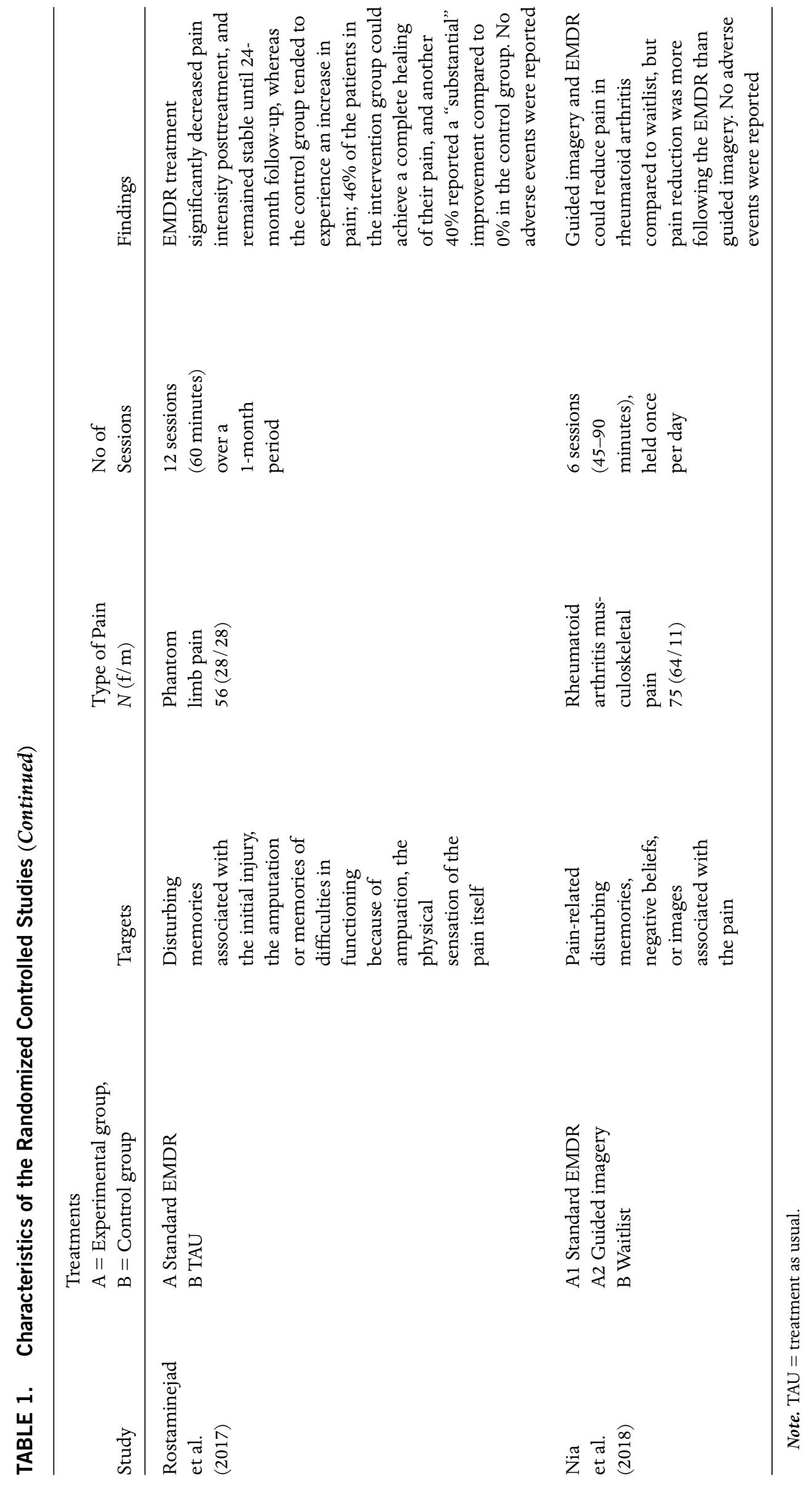




\section{Summary of the Randomized Controlled Trials}

The efficacy of EMDR in the treatment of chronic pain has so far been investigated in a total of six randomized controlled trials. One of the first randomized trials investigated the effect of a modified EMDR short-term intervention on the course of acute migraine attacks (Marcus, 2008). For this purpose, the influence of an "integrated EMDR" treatment intervention was compared with standard medication. While the EMDR intervention group $(N=26)$ was not allowed to take any pain medication during the observation period, the comparison group $(N=26)$ received its individual acute medication to cope with the migraine attack. Instead of medication, the EMDR intervention group received a single EMDR short-term intervention, which consisted of a combination of diaphragmatic breathing, external rhythmic head compressions, and bilateral eye movements. All participants were treated during the mid to late stages of a migraine attack, and pain conditions were assessed by an independent evaluator before, after, 24 hours, 48 hours, and 7 days after treatment. Both the standard medication and the integrated EMDR treatment groups showed a reduction in migraine pain immediately after treatment and 7 days later. However, the integrated EMDR treatment reduced or eliminated migraine pain more rapidly and showed significantly greater improvements compared to conventional medication. No adverse events were reported. A limitation of this study, which makes comparison with other EMDR studies difficult, is certainly the modified form of EMDR intervention used in this study. This began with the participant's use of diaphragmatic breathing in conjunction with rhythmic head compression carried out by the therapist. After this introductory phase, the therapist stopped the rhythmic head compressions and started a set of bilateral stimulation in the form of slow and fast eye movements in a figure of eight for 30-90 seconds. The entire session usually lasted between 12 and 30 minutes. In this respect, this intervention differs significantly from a classical EMDR therapy, and it remains questionable to what extent these results generalize to classical EMDR therapy.

Another interesting randomized trial is described in the doctoral thesis of the American psychologist Linda Estergard (2009), which examined the effectiveness of a classical EMDR intervention on pain symptoms in pain patients for the first time. In this thesis, which unfortunately has not yet been published in a peer-reviewed journal, the efficacy of six sessions of EMDR was compared with a treatment as usual waitlist control in a coordinated two-group design. The participants were matched to their baseline pain levels and randomized to EMDR or a waiting list group with delayed treatment. The experimental group received six 90-minute EMDR sessions over a period of 6 weeks, and the control group waited. The patient population and EMDR treatment are unfortunately not described in more detail in the article. The results showed that EMDR was significantly superior to the waiting list in terms of reducing pain and improving dysphoria. This study is of particular importance for two reasons: Firstly, this study showed the efficacy of the classic EMDR protocol in pain patients for the first time, and secondly, the effects with an effect size of hedges $\mathrm{g}=1.12[-1.82,-0.42]$ can be considered as clinically significant. One weakness is certainly that the work has not yet been formally published, and therefore relevant information (such as type of pain, age of patients, or therapy targets) remains unclear.

In the authors' own study, patients with nonspecific chronic back pain who reported previous experiences with psychological trauma were randomized to standardized pain-focused EMDR in addition to routine care or routine care alone (control; Gerhardt et al., 2016). The results of this RCT showed that pain intensity decreased significantly after EMDR treatment and effects remained stable at 6-month follow-up. On an individual patient basis, the analysis showed that about $50 \%$ of the patients in the intervention group had clinically relevant improvements and assessed their situation as clinically satisfactory compared to no patients in the control group. Notably, secondary analyses of the healthcare utilization behavior during the study period showed that patients in the control group had on average twice as many additional appointments with healthcare providers than patients in the EMDR intervention group, indicating significant cost effectiveness. However, it must also be mentioned that this study was designed as a feasibility study with small sample size, and was therefore not sufficiently powered for confirmatory decisions about efficacy of EMDR. Furthermore, EMDR was not compared to other psychotherapeutic treatments in this study but compared to a wait-list control group. It has been noted that such designs may overestimate intervention effects, thus results might be biased. 
To avoid this limitation, Nia et al. (2018) conducted a three-arm study in patients with chronic musculoskeletal pain due to rheumatoid arthritis. In this randomized controlled trial, 75 pain patients were allocated into two intervention groups and one treatment-as-usual control group. EMDR and guided imagery were provided in an intensive manner, with six daily sessions of 45-90 minutes. Results showed that EMDR (in addition to multimodal rehabilitation treatment) was significantly superior not only to multimodal rehabilitation treatment alone, but also to guided imagery. Guided imagery and EMDR both reduced pain in rheumatoid arthritis over and above the multimodal rehabilitation treatment, but pain reduction was larger following EMDR than guided imagery.

Particularly remarkable results were reported from a randomized controlled trial on the efficacy of EMDR on phantom limb pain in patients after limb amputations (Rostaminejad et al., 2017). In this study, a total of 60 patients with amputations were allocated randomly into experimental or control group. Control group patients were allocated to routine care, whereas the EMDR group received 12 1-hour sessions distributed over a period of 1 month. The mean pain intensity decreased in the experimental group between the first and last session, whereas the control group tended to experience an increase in pain. The differences were statistically significant and clinically highly relevant. In the 2-year follow-up period, over $86 \%$ of patients in the EMDR intervention group reported a complete or almost complete pain relief with just 12 sessions of EMDR therapy-compared to $0 \%$ in the control group. These impressive results are impaired by several methodological biases, that is, a lack of information on statistical, procedural, and therapeutic details, which make it hard to evaluate the quality of the data.

Interestingly, there is also preliminary evidence that EMDR is effective in acute pain conditions. Maroufi et al. (2016) explored the effect of a single session of EMDR for acute postoperative pain management in adolescents. The majority of patients reported at least moderate levels of pain postsurgery, despite being on medication. In this randomized controlled study, 56 adolescent surgical patients were allocated to EMDR treatment or a non-EMDR (control) procedure in which patients underwent a 60minute "neutral" interview. Following EMDR treatment, there was a significant decrease in pain intensity, while the control group reported slightly higher (though nonsignificant) levels of pain on average. The fact that a rather relaxing (EMDR) and a potentially stressful (60-minute interview) procedure were compared makes it difficult to evaluate the results in respect to the natural course of postsurgical pain. These results suggest that EMDR may be an effective treatment modality for postoperative pain.

\section{Discussion}

In summary, the use of EMDR in the treatment of pain is still very much in its infancy. At the same time, it must also be emphasized that with a total of six randomized controlled trials, there is now considerable evidence that EMDR appears to be effective in the treatment of pain. However, the studies are generally characterized by lack of active control groups, monocentric design, and small sample size, and usually conducted by very experienced and specialized institutions - so that it remains particularly unclear to what extent the sometimes very impressive results can be transferred to broader application in general clinical use. Thus, there is currently an urgent need for a large multicenter study with a sufficient number of cases and a suitable comparison group.

In addition to conducting methodologically robust clinical studies, the focus of future research should also be on a better understanding of the underlying mechanisms. At present, it has not been conclusively clarified which mechanisms EMDR uses to influence pain. However, a better understanding of the underlying mechanisms of action could also lead to important implications for clinical application. At present, many questions remain unanswered: Which therapy targets should be focused on in pain patients? In which order should the targets be processed? For which group of pain patients is EMDR more suitable and for which not? Which form of EMDR protocol should be used? The current state of the studies does not provide a clear answer to these questions.

\section{Types of Pain Treated With EMDR and Treatment Targets}

It is astonishing that EMDR appears to be effective for a wide variety of pain disorders, regardless of the underlying cause. For specific pain syndromes such as phantom, postoperative, or inflammatory pain, good efficacy of EMDR has been demonstrated as well as for nonspecific pain conditions. Similarly, there is preliminary evidence for the efficacy of EMDR in both chronic pain and acute pain. These results suggest that it is not so much the original origin of the pain that is important for the treatment response as the 
association of pain symptoms with stressful memories, thoughts, or feelings.

Much more important than the question of the type of pain seems to be the question of whether the pain must be related to psychological traumatization or whether the pain itself is not a kind of "trauma" that can be processed "trauma-therapeutically" with the help of EMDR. The current state of studies seems to indicate that both is possible. Most studies used the standard EMDR protocol to focus on the processing of traumatic memories associated with pain (De Roos et al., 2010; Gerhardt et al., 2016; Grant \& Threlfo, 2002; Konuk et al., 2011; Maroufi et al., 2016; Mazzola et al., 2008; Wilensky, 2006) or traumatic memories completely independent of the pain (Gerhardt et al., 2016), or on possible pain triggers (Gerhardt et al., 2016; Maroufi et al., 2016; Schneider et al., 2008). In addition, numerous studies (De Roos et al., 2010; Grant \& Threlfo, 2002; Kavakci, Semiz, Kaptanoglu, \& Ozer, 2012; Marcus, 2008; Mazzola et al., 2008) have reported that the pain itself can be targeted and processed by using modified "pain-specific" protocols. In such protocols, special attention is often paid to the processing of pain-associated fears, as well as to enabling changes in pain sensations and developing new coping strategies based on these changes. Overall, however, the current data situation does not allow any conclusions to be drawn as to whether one approach is superior to another. However, it can be assumed that different patient groups will benefit from different approaches, and it will be the challenge of future studies to test which approach is best for which patient group.

\section{Clinical Implications and Outlook for Future Research}

Despite increasing scientific evidence, many questions remain regarding the practical use of EMDR in patients with pain. This concerns in particular the question of the selection and modification of "special EMDR pain protocols." At present no clear statements can be made regarding the most favorable approach in the selection of protocols. Preliminary evidence suggests that EMDR in the treatment of chronic pain may require a greater variety of goals and more flexibility in protocol application than the treatment of posttraumatic stress disorder. In addition, the exploratory description of the course of most studies suggests that the goals for desensitization and reprocessing should not be limited to pain or traumatic life events in advance, but should be adapted to the individual as needed. It will be the task of future studies to create more clarity in this area and thus perhaps to make the therapy protocols even more efficient and patientcentered.

\section{Conclusions}

In summary, it can be stated that the consistent findings across the existing studies show a good to very good efficacy of EMDR in the treatment of chronic pain. In recent years, several randomized controlled trials with sufficient sample size have been completed that support the positive effects of EMDR for different pain conditions. However, caution is needed when interpreting the results as these studies comprised different pain conditions and EMDR protocols (including varying numbers of sessions) as well as methodological limitations (see Table 1). Large multicenter studies, which test the use of EMDR in pain therapy outside specific centers, are lacking to date and are an important area for further work.

\section{References}

Allen, T. M. (2004). Efficacy of EMDR and chronic pain management. Chicago, IL: Argosy University.

Buchbinder, R., Van Tulder, M., Öberg, B., Costa, L. M., Woolf, A., Schoene, M., ... Croft, P. (2018). Low back pain: A call for action. Lancet, 391(10137), 2384-2388. doi:10.1016/S0140-6736(18)30488-4

De Roos, C., Veenstra, A. C., De Jongh, A., Den HollanderGijsman, M., Van der Wee, N. J., Zitman, F. G., ... Van Rood, Y. R. (2010). Treatment of chronic phantom limb pain using a trauma-focused psychological approach. Pain Research Management, 15(2), 65-71. doi:10.1155/2010/981634

Estergard, L. (2009). Eye movement desensitization and reprocessing in the treatment of chronic pain (Unpublished doctoral dissertation). Minneapolis, MN: Walden University.

GBD 2016 Disease and Injury Incidence and Prevalence Collaborators. (2017). Global, regional, and national incidence, prevalence, and years lived with disability for 328 diseases and injuries for 195 countries, 19902016: A systematic analysis for the Global Burden of Disease Study 2016. Lancet, 390(10100), 1211-1259. doi:10.1016/S0140-6736(17)32154-2

Gerhardt, A., Leisner, S., Hartmann, M., Janke, S., Seidler, G. H., Eich, W., \& Tesarz, J. (2016). Eye movement desensitization and reprocessing vs. treatment-asusual for non-specific chronic back pain patients with psychological trauma: A randomized controlled pilot study. Frontiers in Psychiatry, 7(201), 1-10. doi:10.3389/ fpsyt.2016.00201 
Grant, M., \& Threlfo, C. (2002). EMDR in the treatment of chronic pain. Journal of Clinical Psychology, 58(12), 15051520. doi:10.1002/jclp.10101

Hashmi, J., Baliki, M., Huang, L., Baria, A., Torbey, S., Hermann, K., \& . . Apkarian, A. (2013). Shape-shifting pain: Chronification of back pain shifts brain representation from nociceptive to emotional circuits. Brain, 136(9), 2751-2768. doi:10.1093/brain/awt211

International Association for the Study of Pain. (2012). Unrelieved pain is a major global health-care problem. Washington, DC: International Association for the Study of Pain.

Kavakci, O., Semiz, M., Kaptanoglu, E., \& Ozer, Z. (2012). EMDR treatment in fi bromyalgia, a study of seven cases. Anatolian Journal of Psychiatry, 13, 75-81.

Konuk, E., Epözdemir, H., Atçeken, Ş. H., Aydin, Y. E., \& Yurtsever, A. (2011). EMDR treatment of migraine. Journal of EMDR Practice and Research, 5(4), 166-176. doi:10.1891/1933-3196.5.4.166

Marcus, S. V. (2008). Phase 1 of integrated EMDR: An abortive treatment for migraine headaches. Journal of EMDR Practice and Research, 2(1), 15-25. doi:10.1891/1933-3196.2.1.15

Maroufi, M., Zamani, S., Izadikhah, Z., Marofi, M., \& O'Connor, P. (2016). Investigating the effect of eye movement desensitization and reprocessing (EMDR) on postoperative pain intensity in adolescents undergoing surgery: A randomized controlled trial. Journal of Advanced Nursing, 72(9), 2207-2217. doi:10.1111/jan.12985

Mazzola, A., Calcagno, M. L., Goicochea, M. T., Pueyrredòn, H., Leston, J., \& Salvat, F. (2009). EMDR in the treatment of chronic pain. Journal of EMDR Practice and Research, 3(2), 66-79. doi:10.1891/1933-3196.3.2.66

Nia, N. G., Afrasiabifar, A., \& Behnammoghadam, M. (2018). Comparing the effect of eye movement desensitization and reprocessing (EMDR) with guided imagery on pain severity in patients with rheumatoid arthritis. Journal of Pain Research, 28(11), 2107-2113. doi:10.2147/JPR.S158981

Rostaminejad, A., Behnammoghadam, M., Rostaminejad, M., Behnammoghadam, Z., \& Bashti, S. (2017). Efficacy of eye movement desensitization and reprocessing on the phantom limb pain of patients with amputations within a 24-month follow-up. International Journal of Rehabilitation Research, 40(3), 209-214. doi:10.1097/MRR.0000000000000227
Schneider, J., Hofmann, A., Rost, C., \& Shapiro, F. (2008). EMDR in the treatment of chronic phantom limb pain. Pain Medicine, 9(1), 76-82. doi:10.1111/j.15264637.2007.00299.x

Tesarz, J., Gerhardt, A., Leisner, S., Janke, S., Treede, R. D., \& Eich, W. (2015). Distinct quantitative sensory testing profiles in nonspecific chronic back pain subjects with and without psychological trauma. PAIN, 156(4), 577586. doi:10.1097/01.j.pain.0000460350.30707.8d

Tesarz, J., Leisner, S., Gerhardt, A., Janke, S., Seidler, G., Eich, W., \& Hartmann, M. (2014). Effects of eye movement desensitization and reprocessing (EMDR) treatment in chronic pain patients: A systematic review. Pain Medicine, 15(2), 247-263. doi:10.1111/pme.12303

Tesarz, J., Seidler, G. H., \& Eich, W. (2015). Treatment of pain with EMDR (in German). Stuttgart: Klett-Cotta.

Tesarz, J., Wolfgang, E., Treede, R. D., \& Gerhardt, A. (2016). Altered pressure pain thresholds and increased wind-up in adult chronic back pain patients with a history of childhood maltreatment: A quantitative sensory testing study. PAIN, 157(8), 1799-1809. doi: $10.1097 /$ j.pain.0000000000000586

Wilensky, M. (2006). Eye movement desensitization and reprocessing (EMDR) as a treatment for phantom limb pain. Journal of Brief Therapy, 5(1), 31-44.

Williams, A. C., Eccleston, C., \& Morley, S. (2012). Psychological therapies for the management of chronic pain (excluding headache) in adults. Cochrane Database Systematic Reviews, 11, CD007407. doi:10.1002/14651858.CD007407.pub3

Disclosure. J.T. receives income from published books on EMDR therapy and for the training of licensed professionals in EMDR therapy. G.H.S. receives income from book publications on EMDR and training licensed professionals in this approach. The other authors have no relevant financial interest or affiliations with any commercial interests related to the subjects discussed within this article.

Correspondence regarding this article should be directed to Jonas Tesarz, Department of General Internal Medicine and Psychosomatics, University Hospital Heidelberg, Heidelberg, Germany, INF 410, 69120. E-mail: jonas.tesarz @)med.uni-heidelberg.de 